\title{
AN APPLICATION OF THE SPECIAL REISSNER-NORDSTRÖM SPACE-TIME TO DESCRIPTION OF THE UNIVERSE IN THE NEIGHBOURHOOD OF THE PLANCK ERA
}

Abstract. The description of the special Reissner-Nordström space-time in the Planck era is presented and its comparison with the Robertson-Walker space-time is given.

1. Introduction. The aim of this paper is to present the conjectural description of Universe by means of a special 4-dimensional Reissner-Nordström (briefly R-N) space-time and to compare it with the description given by the 4-dimensional Robertson-Walker (R-W) space-time. Let us recall that according to one of the cosmological conjectures the history of Universe began with Big Bang [6]. We will start our description at time $t_{1}=10^{-44}$ sec, i.e. at the end of the Planck era, and conclude it at time $t_{2}=10^{-34} \mathrm{sec}[6]$.

Definition 1. The generalized 4-dimensional $R-N$ space-time is defined by the metric tensor

$$
\operatorname{diag}\left(-E^{a+1}, E^{a-1}, r^{2}, r^{2} \sin ^{2} \theta\right)
$$

where

$$
\begin{gathered}
E=1-\frac{r_{0}}{r}+\frac{K r_{0}^{2}}{r^{2}}, \quad a \in[0,1], \\
K \in\left(\frac{1}{4}, \frac{9}{32}\right), \quad r_{0}=\text { const }>0 \\
\left(x^{1}, x^{2}, x^{3}, x^{4}\right)=(t, r, \theta, \varphi) .
\end{gathered}
$$

2000 Mathematics Subject Classification: 53B20, 53B50.

Key words and phrases: Reissner-Nordström space-time, Robertson-Walker spacetime, inflation model. 
For $a=1$ the metric tensor (1) has the form

$$
\operatorname{diag}\left(-E^{2}, 1, r^{2}, r^{2} \sin ^{2} \theta\right)
$$

Definition 2. The special $R-N$ space-time is one with metric tensor (2) and parameter $K=(1+\varepsilon) / 4$, where $\varepsilon>0$ is close to zero.

Diagram (9) of [4] shows the graph of scalar curvatures $T=T(r)$ in the model of 4-dimensional generalized R-N space-time in dependance on the radius $r$ of the Universe. We will determine the radii $r^{\prime}$ and $r^{\prime \prime}$ for the scalar curvatures $T_{1}, T_{2}$ corresponding to $t_{1}$ and $t_{2}$. In [4] the scalar curvature $T_{\varepsilon}$ of the special R-N space-time $(a=1)$ was determined in the form

$$
T_{\varepsilon}=\frac{4(1+\varepsilon) r_{0}^{2}}{r^{2}\left[4\left(r-\frac{r_{0}}{2}\right)^{2}+\varepsilon r_{0}^{2}\right]}
$$

or equivalently

$$
r^{2}-\frac{r_{0}}{2} r-\frac{r_{0}}{\sqrt{T_{\varepsilon}}}=0
$$

The solutions of (4) are

$$
\widehat{r}_{1}=\frac{r_{0}}{4}-\frac{1}{2} \sqrt{\frac{r_{0}^{2}}{4}+\frac{4 r_{0}}{\sqrt{T_{\varepsilon}}}}, \quad \widehat{r}_{2}=\frac{r_{0}}{4}+\frac{1}{2} \sqrt{\frac{r_{0}^{2}}{4}+\frac{4 r_{0}}{\sqrt{T_{\varepsilon}}}} .
$$

It follows from (3) that

$$
\lim _{r \rightarrow 0^{+}} T=+\infty, \quad \lim _{\substack{r \rightarrow r_{0} / 2 \\ \varepsilon \rightarrow 0^{+}}} T=+\infty,
$$

so we can assume, for $\varepsilon$ close to zero, that $4 r_{0} / \sqrt{T_{\varepsilon}}=0$.

The radius $r$ is close to zero so the approximation $\sqrt{x} \approx \frac{1}{2} x$ allows one to obtain the following values:

$$
r^{\prime}=\frac{r_{0}}{4}-\frac{r_{0}^{2}}{16}, \quad r^{\prime \prime}=\frac{r_{0}}{4}+\frac{r_{0}^{2}}{16} .
$$

The above data are illustrated in diagram (7) below.

We can summarize this in the following theorem.

THEOREM 1. In the special 4-dimensional $R-N$ space-time with metric tensor (2) there exist two Big Bangs for $r=0$ and $r=r_{0} / 2$ given in dia$\operatorname{gram}(7)$. 


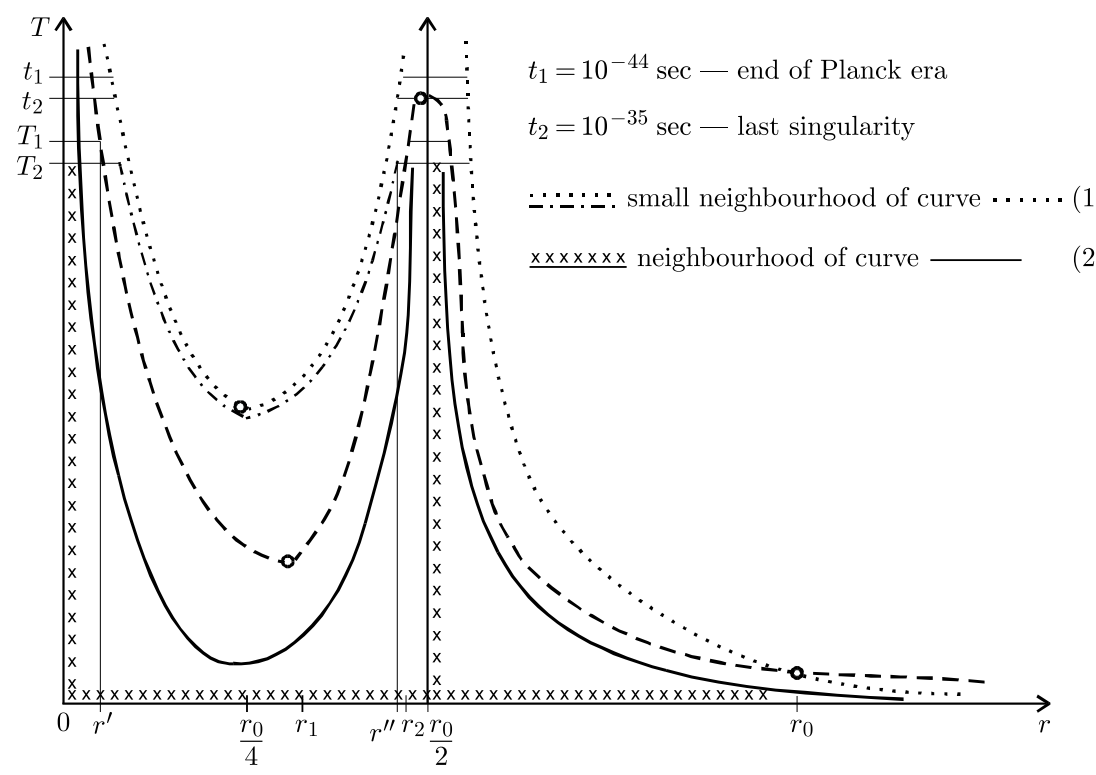

Definition 3. The 4-dimensional Robertson-Walker (R-W) space-time is defined by the following metric tensor $([2$, p. 190]):

$$
\operatorname{diag}\left(-1, \frac{R^{2}}{1-k r^{2}}, R^{2} r^{2}, R^{2} r^{2} \sin ^{2} \theta\right),
$$

where $R=R(t)$ denotes the dimension free scale factor and $k \in[-1,1]$ is a parameter related with space curvature.

There are three Friedmann models of this space-time [2] described as follows:

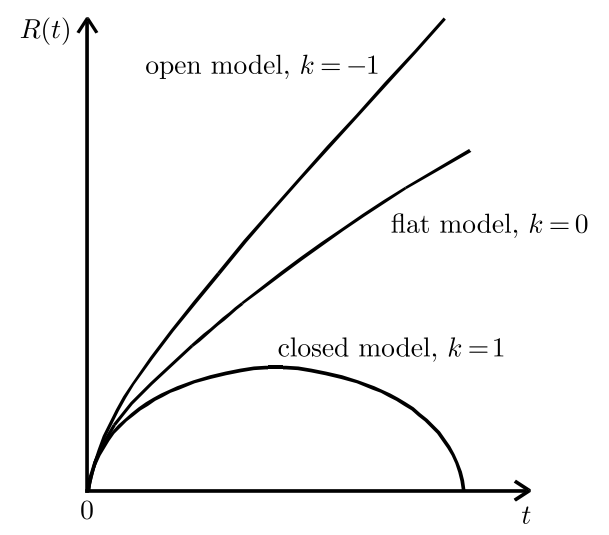

We are interested in the flat Friedmann model $(k=0)$. In this model the 
scale factor $R(t)$ has the form $[2]$

$$
R(t)=\left(\frac{3 A}{2}\right)^{2 / 3} t^{2 / 3}, \quad A>0, \quad A=8 \pi G \varrho_{0} R_{0}^{3} / 3
$$

and inflation occurs.

The inflation model can be described by the diagram [1]

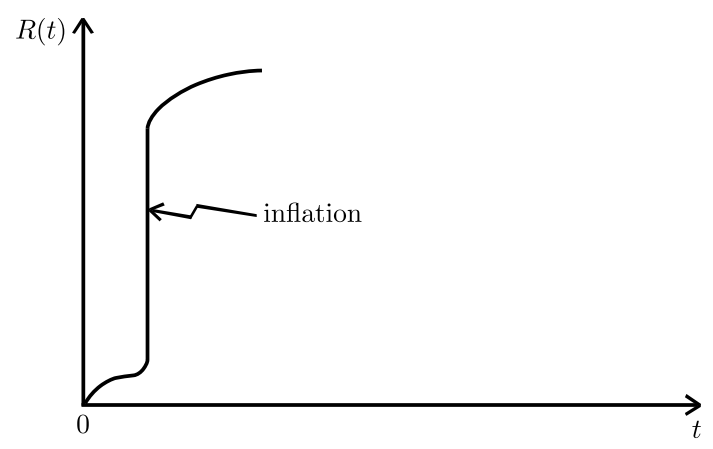

In the inflation model, immediately after Big Bang there occurs a rapid and impetuous growth of the Universe. The inflation process ends at time $t_{2}=10^{-34} \sec [6]$.

THEOREM 2. In the flat model of the $R$ - $W$ space-time with metric tensor (8) there occurs a Big Bang for $r=0$ and inflation for $r=r_{0} / 2$.

Corollary 1 . In the flat model of the $R$ - $W$ space-time inflation occurs exactly at the moment when the second Big Bang occurs in the special $R-N$ space-time.

COROLlary 2. The graph of the scalar curvature of the special $R-N$ space-time is the curve (1) in diagram (7).

Observe that in the generalized R-N space-time the graph of the scalar curvature tends for $a \rightarrow 0^{+}$to the limit curvature $T=0$ for $r \neq r_{0} / 2$ and to $T=+\infty$ for $r=r_{0} / 2$ (see (2) in diagram (7)).

In $[3$, p. $223,(2.14)]$ we have presented a formula for the Weyl curvature. Now we can give the following complement.

Corollary 3. The neighbourhood of a Big Bang or the neighbourhood of a Black Hole has for $0<a<1$ the structure of a generalized $R$ - $N$ space-time (see (2) in diagram (7)).

REMARK. It follows from the above corollaries that in the model of the Universe described by the flat R-W space-time, inflation occurs at time $t_{2}$ while in the model described by the special R-N space-time the second Big Bang occurs. 
It is a problem of experimental cosmology to investigate which of these conjectures is valid [5].

\section{References}

[1] P. Davies, The Last Three Minutes, Orion Publ., 1994.

[2] J. Foster and J. D. Nightingale, A Short Course in General Relativity, Longman, 1979.

[3] B. Glanc and A. Jakubowicz, Application of the Weyl curvature tensor to description of the generalized Reissner-Nordström space-time, Appl. Math. (Warsaw) 27 (2000), 219-223.

[4] B. Glanc, A. Jakubowicz and H. Kleczewska, The generalized Reissner-Nordström space-time, Demonstratio Math. 30 (1997), 635-639.

[5] A. H. Guth, The Inflationary Universe, Jonathan Cape, 1997.

[6] A. Liddle, An Introduction to Modern Cosmology, Wiley, 1999.

Institute of Mathematics

Technical University of Szczecin

Al. Piastów 17

70-310 Szczecin, Poland

E-mail: glanc@arcadia.tuniv.szczecin.pl 